\title{
NOTA CRÍTICA SOBRE LA GENERALIDAD ABSOLUTA
}

\author{
Agustín Rayo y Gabriel Uzquiano Cruz (comps.), Absolute \\ Generality, Oxford University Press, Oxford, 2006, 396 pp.
}

\author{
Eduardo Alejandro Barrio \\ CONICET \\ Universidad de Buenos Aires \\ Grupo de Acción Filosófica (GAF) \\ ebarrio@fibertel.com.ar
}

Si no fuera posible desarrollar instrumentos lingüísticos cuyos cuantificadores carecieran de toda restricción, descubriríamos un nuevo resultado limitativo. Así como bajo ciertas condiciones somos incapaces de expresar dentro de nuestros lenguajes sus propios conceptos semánticos, ${ }^{1}$ si los cuantificadores siempre fueran restrictos, tales instrumentos carecerían de la posibilidad de hablar sobre absolutamente todo lo que hay. Por ejemplo, al decir:

Todo es idéntico a sí mismo

o

Nada existe y subsiste

o al comprometernos con la tesis según la cual

No hay objetos abstractos

seríamos incapaces de hablar acerca de la totalidad de las entidades del universo, sin ninguna restricción. Absolute Generality, compilado por Agustín Rayo y Gabriel Uzquiano, es más que una recopilación de artículos que reúne a gran parte de los principales especialistas

${ }^{1}$ De acuerdo con los trabajos de Tarski, la autorreferencia y la universalidad semántica implican, razonando clásicamente, paradojas que impiden, básicamente, dar una definición correcta de la verdad dentro del lenguaje (Tarski 1935). 
del área. ${ }^{2}$ Al reconocer el potencial de un tema generador de una discusión fundamental, los compiladores brindan un espacio para el surgimiento de algunos resultados importantes ligados a los medios expresivos de los lenguajes, su semántica y el uso de los cuantificadores. Seguramente este libro se convertirá en referencia obligatoria para los futuros trabajos del área. Los vínculos entre la teoría de modelos y la teoría de conjuntos, entre el Teorema de Tarski y la capacidad expresiva de los cuantificadores, el recurso a dominios de cuantificación y la posibilidad de diagonalizar sobre ellos, la cuantificación plural y los recursos a los lenguajes de orden superior son algunos de los temas que se abordan con profundidad y crean un entramado detallado que ayuda a ubicarse dentro del laberinto de los estudios semánticos contemporáneos.

El objetivo central de esta antología es estudiar los diferentes problemas vinculados a la generalidad absoluta, esto es, a la posibilidad de cuantificar sobre todo lo que hay brindando los argumentos más importantes a favor y en contra de tal posibilidad. Absolute Generality plantea las diferentes estrategias para resolver problemas de limitación expresiva que se articulan en los distintos trabajos allí presentados. En esta dirección, Kit Fine desarrolla el uso de un nuevo tipo de operador modal para resolver diferentes limitaciones expresivas vinculadas con la extensibilidad indefinida de los dominios. Michael Glanzberg, Geoffrey Hellman y Charles Parsons adoptan distintas versiones de contextualismos para analizar los cuantificadores. Parsons también los vincula con las expresiones sistemáticamente ambiguas (systematically ambiguous). Shaugham Lavine, en cambio, incorpora sus ideas sobre esquemas (full schemas). Agustín Rayo explora la jerarquía de cuantificadores de orden superior y sus posibilidades expresivas. Øystein Linnebo incorpora propiedades expandiendo los compromisos conjuntistas usuales para obtener máxima generalidad. Alan Weir recurre a los enfoques kripkeanos en el marco de los lenguajes que pueden expresar su propia semántica debilitando la lógica clásica. Vann McGee apela a la aplicación absolutamente irrestricta de las reglas de introducción y de eliminación de los cuantificadores. En la misma dirección, y también vinculando la aplicación abierta (open-ended) de las reglas de inferencia y, en

${ }^{2}$ El libro ha sido reseñado por Priest (2007), Dieveney (2008) y Smith (2008). También hay comentarios sobre él en el blog del propio Peter Smith, 2007, “Absolute Generality", Logic Matters, <http://logicmatters.blogspot.com/2007/07/absolute-generality-l-kit-fine-and-all.html > [5/08/2007], y en el de Brian Weatherson, 2005, "Universalism Qualified", Thoughts Arguments and Rants, <http://tar. weatherson.org/> [5/01/2005]. 
general, de las expresiones lógicas, Timothy Williamson explora los vínculos entre absoluta identidad y absoluta generalidad. Gabriel Uzquiano presenta y analiza ciertos problemas internos del absolutismo: nuestras mejores teorías formales parecen condicionar el "tamaño" del universo de manera tal que el mismo resulte incompatible con la absoluta generalidad. Finalmente, el trabajo de Stewart Shapiro y Crispin Wright estudia a fondo las consecuencias, dentro de la filosofía de las matemáticas, producto de la existencia de totalidades indefinidamente extensibles (indefinitely extensible totalities).

Además, en la introducción, Rayo y Uzquiano exponen de manera clara y concisa el estado de la cuestión en torno a la posibilidad de cuantificar sobre absolutamente todo; ahí ofrecen una efectiva puerta de entrada al debate para todos los que desconocen en detalle los inicios del mismo. Hacen una descripción completa del panorama que abarca desde las posiciones de los más fervientes defensores de la cuantificación irrestricta, hasta las de sus enemigos más combativos. Dicha descripción se inicia con el reconocimiento de que hablar de generalidad absoluta supone dos preguntas distintas:

¿Hay un dominio universal (all-inclusive) para los cuantificadores de nuestros lenguajes?

Y más allá de toda cuestión metafísica,

¿Están nuestros lenguajes preparados para expresar generalizaciones acerca de ese dominio?

Por supuesto, tal como lo reconocen los compiladores, ambas son cuestiones al menos parcialmente independientes. A continuación, Rayo y Uzquiano presentan a los principales actores de esta trama: los defensores de la generalidad absoluta (o absolutistas), quienes sostienen que hay un dominio universal y que nuestros cuantificadores son capaces de expresar, cuando menos en ciertas circunstancias, semejante generalidad, y sus detractores (o antiabsolutistas), quienes basados en ciertos argumentos escépticos rechazan la existencia de un dominio universal o el carácter irrestricto de los cuantificadores. Fine, Glanzberg, Hellman y Lavine representan, en el libro, al grupo antiabsolutista, mientras que Linnebo, McGee, Weir y Williamson forman parte de los absolutistas. Rayo, Shapiro, Uzquiano y Wright exploran los matices entre ambos grupos. Luego, la introducción reconstruye los principales argumentos escépticos acerca de la posibilidad de tener cuantificación absolutamente irrestricta. Para adoptar 
una posición sobre la absoluta generalidad, es menester valorar algunas cuestiones que se resumen en las siguientes preguntas: ¿son siempre los dominios de cuantificación extensibles de manera indefinida?, o ¿debemos comprometernos con algún tipo de objeto colectivo como dominio de cuantificación?, o ¿̨hay siempre algún tipo de restricción sortal para nuestra cuantificación?, o incluso ¿es siempre la ontología dependiente de un marco conceptual o teoría de tal manera que nunca podremos cuantificar sobre absolutamente todo?, o ¿hay indeterminaciones semánticas vinculadas a los lenguajes formales (tales como el teorema Löwenheim-Skolem) que nos hagan dudar de la posibilidad de hablar de todo? Finalmente, la introducción incluye un breve resumen de cada uno de los artículos editados en el libro y una bibliografía completa.

El proyecto de desarrollar un lenguaje $L$ en el que se pudiera hablar de todo lo que hay requerirá que podamos hablar incluso de las interpretaciones de las expresiones de $L$. Más aún, las propiedades lógicas de sus expresiones, tales como los vínculos inferenciales entre sus oraciones, dependen de la totalidad de las interpretaciones. Por eso, que seamos capaces de hablar de absolutamente todas las interpretaciones de las expresiones no lógicas de $L$ parece ser una condición que nuestra teoría de la interpretación de los lenguajes tiene que cumplir. Resulta que necesitamos que exista la unión de todos los dominios de todas las interpretaciones, a la vez que necesitamos emplear la cuantificación absolutamente irrestricta al dar la semántica de los lenguajes formales. Sin embargo, haciendo algunas suposiciones naturales acerca de las semánticas de los lenguajes formales, y en ausencia del conjunto de todos los conjuntos, ninguna interpretación de los cuantificadores del lenguaje parece lograr absoluta generalidad. Toda vez que se fije un rango determinado para los cuantificadores de un lenguaje, este procedimiento nos habilitará para definir un nuevo objeto que no esté bajo el alcance de los cuantificadores del lenguaje y establecerá que la cuantificación no era irrestricta en absoluto.

Supongamos que $L$ es un lenguaje de primer orden cuyos cuantificadores son interpretados de manera irrestricta. Sea $P$ un predicado monádico de $L$. Sea $F$ cualquier predicado significativo del metalenguaje. Según Williamson, debe ser posible interpretar $P$ como si significara $F$. Esto es,

(1) $\forall x\left(i_{F}\right.$ es una interpretación bajo la cual $P$ se aplica a $x$ ssi $\left.F x\right)$. 
Por ejemplo, si el metalenguaje es el español, un caso de este tipo consiste en " $\forall x\left(i_{F}\right.$ es una interpretación bajo la cual $P$ se aplica a $x$ ssi $x$ es un hombre)", donde " $\forall x$ " tiene un rango irrestricto. Entonces, dada la suposición de que las interpretaciones son objetos, parece razonable permitir definir un nuevo predicado $R$ tal que:

(2) $\forall x(R x \operatorname{ssi} x$ no es una interpretación bajo la cual $P$ se aplica a $x)$.

Si en (1), $R$ reemplaza a $F$ y, al mismo tiempo, aplicamos la definición de $R$ en (2), obtenemos:

(3) $\forall x\left(i_{R}\right.$ es una interpretación bajo la cual $P$ se aplica a $x$ ssi $x$ no es una interpretación bajo la cual $P$ se aplica a $x$ ).

Ya que " $\forall x$ " en (3) es absolutamente irrestricto, podemos instanciarlo y obtener:

(4) $i_{R}$ es una interpretación bajo la cual $P$ se aplica a $i_{R}$ ssi $i_{R}$ no es una interpretación bajo la cual $P$ se aplica a $i_{R}$.

El argumento parece indicar algún tipo de limitación a los recursos expresivos del metalenguaje en el que presuntamente hablamos de todas las interpretaciones: nunca seremos capaces de cuantificar sobre absolutamente todo si las interpretaciones caen bajo el alcance de sus cuantificadores. Esto es, hay atribuciones posibles de significados a las oraciones de $L$ a las que no les corresponde ninguna interpretación que pueda estar en el rango de los cuantificadores de un lenguaje cuyos cuantificadores pueden hablar de todo. ${ }^{3}$ Los trabajos de Glanzberg, Lavine, Linnebo y Parsons, reunidos en Absolute Generality, presentan y discuten distintas versiones de una versión de la paradoja de Russell planteada por Williamson (2003). Si bien el artículo de Rayo no analiza directamente el argumento de Williamson, se puede leer como el resultado de asumir sus consecuencias. ${ }^{4}$ Todos ellos discuten y enfrentan las dificultades que acarrea la posibilidad de un lenguaje que posea cuantificación irrestricta en el que se pueda hablar de toda interpretación.

${ }^{3}$ Un punto importante es que el argumento de Williamson implica una contradicción que no depende de que, a diferencia de lo que aparentemente sucede con la Paradoja de Orayen, las interpretaciones sean conjuntos. Para apreciar el planteamiento, véase Orayen 2003; en García de la Sienra 2008 se puede encontrar parte del desarrollo de la discusión posterior.

${ }^{4}$ Rayo analiza y discute el argumento en Rayo 2005. 
El argumento supone que las interpretaciones de las expresiones no lógicas del lenguaje son objetos (SEM1); que no hay ninguna restricción para la formación de nombres y predicados en el lenguaje (SEM2); que es posible cuantificar sobre todo lo que hay (SEM3); que la lógica aceptada debe ser la clásica (SEM4); y que el lenguaje es semánticamente autosuficiente: debe tener suficiente poder expresivo para formular en su interior su propia teoría de la interpretación (SEM5). En la compilación, Linnebo, Rayo y Weir intentan responder al argumento sugiriendo distintas estrategias a partir de las cuales sea posible desarrollar consistentemente la semántica de un lenguaje en un contexto en el cual los cuantificadores del lenguaje son absolutamente irrestrictos. Glanzberg y Parsons, en cambio, lo usan para limitar los recursos de la cuantificación. En esta línea, en "The Problem of Absolute Universality", Parsons sostiene que lo que prueba el argumento es que ese recurso cuantificacional no es posible y que toda cuantificación es definitivamente restricta. Esto es, negar (SEM3). Una estrategia usual asociada a esta opción indicaría que nociones como interpretación son indefinidamente extensibles (del mismo modo que lo serían nociones como dominio, modelo, conjunto). Esta opción impide tener un lenguaje suficientemente general como para cuantificar sobre todos los objetos. Por esa razón, aceptarla implica aceptar una profunda limitación expresiva: nunca seremos capaces de desarrollar una semántica absolutamente general. Por ese motivo, tal opción implica, además, limitar (SEM5).

Otra estrategia posible, distinta de la anterior, consiste en adoptar algún tipo de respuesta jerárquica vinculada a la cuantificación sobre interpretaciones. Se trata de abandonar el supuesto según el cual las interpretaciones son objetos que caen bajo el alcance de los cuantificadores de primer orden (SEM1). Han seguido este camino el propio Williamson (2003), recurriendo a cuantificación de tipo superior para hablar sobre interpretaciones, y Rayo, en el artículo de esta compilación, usando cuantificación plural. En las respuestas jerárquicas se diferencian dos distintos tipos de cuantificadores, sin que objetos e interpretaciones interactúen, lo que provoca que los cuantificadores de primer orden logren hablar de todos los objetos, mientras que los cuantificadores de orden superior o los plurales permitan expresar las interpretaciones. No es cierto que las interpretaciones sean objetos, ya que ellas son los valores de cuantificadores con mayor poder expresivo que los de primer orden. Sin embargo, una simple modificación al argumento semántico muestra que no basta admitir cuantificación de segundo orden o cuantificación plural: hace falta recurrir a una jerarquía infinita de lenguajes cada vez más expresivos. 
No hay ningún lenguaje con recursos expresivos suficientes como para hablar de todas las interpretaciones. Linnebo sostiene que "hay ciertas profundas e interesantes intuiciones que no pueden ser propiamente expresadas"5 y agrega, más adelante, que toda respuesta al argumento que adopte una jerarquía de lenguajes hace imposible que podamos expresar generalizaciones a través de la jerarquía (pp. 154156). En la misma dirección, Rayo sostiene que la presencia de cuantificación absolutamente general es inestable: es imposible brindar una descripción general de las interpretaciones usando cuantificación plural. ${ }^{6}$ El artículo conduce al lector a un trilema: (i) abandonar la cuantificación absolutamente general; (ii) dar una semántica general para ciertos lenguajes es una tarea que está más allá de nuestros capacidades; (iii) adoptar algún tipo de jerarquías sin techo, motivadas tanto en el enfoque tarskiano como en el universo conjuntista de $Z F$.

Las respuestas jerárquicas presentan una nueva limitación expresiva. Ningún lenguaje será semánticamente autosuficiente (SEM5), no sólo en el sentido de ser capaz de expresar su propio predicado veritativo, sino en el de ser capaz de expresar sus propios recursos lógicos para garantizar que sus cuantificadores sean completamente generales. Y lo que puede resultar peor aún: si ninguna variable puede tener como rango más de un nivel de la jerarquía, hay límites a lo que se puede expresar en semántica, ya que desde ningún punto de la jerarquía se puede hablar de toda la jerarquía.

Linnebo presenta una alternativa a las respuestas jerárquicas en "Sets, Properties, and Unrestricted Quantification", la cual consiste en abandonar (SEM2). La idea básica respecto del argumento de Williamson es lograr cuantificación irrestricta, admitiendo que las interpretaciones de todas las expresiones suboracionales de $L$ caen bajo el alcance de cuantificadores de primer orden, pero prohibiendo que los predicados russellianos tengan una interpretación. De manera general, lo que este enfoque plantea es que, respecto de lo que hay, además de urelementos y conjuntos, tenemos propiedades, las cuales son la interpretación de los predicados. Y ya que el desarrollo de una interpretación absolutamente general involucra cuantificadores sin restricciones, capaces de hablar acerca de la interpretación de los predicados, dentro de este enfoque se propone limitar los predicados admisibles en $L$ para restaurar la consistencia. Linnebo propone un teoría mixta de conjuntos y propiedades (ZFCU). Sus axiomas

${ }^{5}$ Todas las traducciones son mías.

${ }^{6}$ Linnebo, Resnik y Parsons han objetado la posibilidad de entender la cuantificación plural sin recurrir a entidades plurales. Cfr. Linnebo 2003, Resnik 1988. 
contienen las modificaciones necesarias para incorporar propiedades al universo de conjuntos sin caer en inconsistencias. El principal resultado del artículo es mostrar la existencia de una interpretación para $L, \mathrm{ZFCU}_{0}+V^{\epsilon}$, que ofrezca:

(i) una interpretación pretendida para ZFCU,

$\mathrm{y}$

(ii) una prueba de consistencia relativa para ZFCU,

en la cual se pueden expresar generalizaciones irrestrictas sobre interpretaciones.

Sin embargo, me gustaría señalar que la propuesta afronta dificultades semejantes a las del enfoque jerárquico. No es difícil advertir que incumple con la autosuficiencia semántica (SEM5). En la propuesta de Linnebo se incluyen ciertos conceptos propios de la teoría en la que se formula la interpretación del lenguaje. Al igual que lo que sucede con los cuantificadores de orden superior o con los cuantificadores plurales, el enfoque incluye la relación especial de posesión $\eta$, que permite hablar dentro del lenguaje de propiedades como si fueran objetos. No obstante, la teoría tiene limitaciones en poder expresivo. En primer lugar, no se puede expresar con toda su generalidad la relación de posesión $\eta$. El enfoque no puede dejar de involucrar una nueva jerarquía infinita de conjuntos y de propiedades: $e_{0}, e_{1}, e_{2} \ldots$ de tal manera cada una de las cuales sirve como la entidad utilizada como relación de posesión del nivel inmediato inferior. Por eso, la teoría nunca llega ser exhaustiva, esto es, en ningún nivel somos capaces de hablar de la entidad que resulta ser la interpretación de $\eta$, lo cual muestra que la teoría no es capaz de aplicarse al lenguaje en el que se formula, y presenta diferencias entre el concepto general de posesión (el cual no puede expresarse dentro del lenguaje) y los conceptos de posesión expresables en cada uno de los niveles.

Una opción diferente frente al argumento de Williamson sería apartarse de la lógica clásica. Hay diversas maneras de hacerlo. Entre los intentos consumados está el enfoque de puntos fijos de Alan Weir incluido en la antología, y el recurso a los dialetheia de Priest; y entre los que podrían intentarlo, el enfoque de Maudlin (2004) y el paracompleto de Field (2008). En todos los casos se acepta que la interpretación del predicado de Russell es un objeto (SEM1) y que el predicado es parte de lo que podemos expresar en el lenguaje 
(SEM2). Todos estos enfoques interpretan a los predicados por medio de propiedades. Y todos involucran algún tipo de modificación de la lógica clásica (SEM4).

En el artículo de Weir "Is It Too Much to Ask, to Ask for Everything?" se explora esta opción abandonando (SEM4). El autor propone un modelo de tipo kripkeano en el cual se intenta lograr cuantificación absolutamente irrestricta, confinando las contradicciones en lo que no es ni verdadero ni falso. Más allá de los detalles, se recurre a un enfoque kripkeano de puntos fijos en el cual la alternativa de contar con afirmaciones cuyo valor queda indeterminado en ese punto libra de la contradicción que parece seguirse del argumento de Williamson sin adoptar una jerarquía ascendente de tipos lógicos ni restricciones al esquema de comprensión naïve. Priest (2007), en su reseña de Absolute Generality, propone una opción paralela a la de Weir. La idea también es abandonar (SEM4). El enfoque recurre a los dialetheia. La contradicción a la que se llega mediante el argumento de Williamson es verdadera y falsa. Una gran virtud de la propuesta de Priest es la capacidad de "absorber" las contradicciones y en particular brindar un modo simple en el cual una teoría podría expresar su propia semántica (Priest 2006). Dada cualquier interpretación, la diagonalización permite la construcción de un objeto que no está en su dominio (trascendencia), pero el objeto está claramente en el dominio de todos los objetos cuantificables, ya que se cuantifica sobre el mismo (closure). ${ }^{7}$ Un camino en alguna medida análogo es el que sigue Hartry Field (2008), quien al modificar las leyes del condicional, evita incluso que del paso (4) del argumento se siga un dialetheia. No me queda claro que el precio de abandonar la clasicidad de la lógica no sea demasiado alto. Tampoco es evidente que estos enfoques logren evitar todo tipo de jerarquías, esto es, que no generen nuevos límites a (SEM5).

\section{III}

Uno de los puntos en discusión en la presente antología es si existe un dominio absolutamente inclusivo. Por supuesto, el recurso a dominios no tiene por qué presuponer que se trata de un objeto conjuntista. Quizá existan colecciones o multiplicidades que obedezcan a principios distintos que los conjuntos. Nótese, además, que la discusión está emparentada con la necesidad de reunir o no en un

${ }^{7}$ En el artículo de Shapiro y Wright incluido en el volumen motivo de esta nota (pp. 271-272), se destacan vínculos entre la extensibilidad indefinida y el inclosure scheme. 
único objeto el dominio de cuantificación, ${ }^{8}$ o con la de reunirlos sin esta suposición. ${ }^{9}$ El artículo de Fine y el de Shapiro y Wright analizan la dificultad de reunir en un único objeto conjuntista el dominio de un cuantificador absolutamente irrestricto. Todos vinculan este problema con el de las totalidades indefinidamente extensibles. Así, recurriendo a las ideas de Dummett, Fine en "Relatively Unrestricted Quantification" explota el clásico argumento de la extensibilidad indefinida (ahora aplicado a los dominios de cuantificación) con el propósito de poner en duda la coherencia de la cuantificación absolutamente general. La versión original del mismo, formulada por Dummett, se aplica a los ordinales:

Tomemos algunos ordinales; entonces, con cualquiera que comencemos, hay una operación la cual nos da un nuevo ordinal (tomemos el sucesor del más grande o si no lo hay tomemos el ordinal límite) [...]. Por lo tanto, no puede haber un dominio determinado que contenga, uno y para siempre, todos los ordinales. (Dummett 1991, p. 316)

Dada cualquier especificación precisa de la totalidad de los números ordinales, siempre se puede ir más allá de esa totalidad; por lo tanto, tener una totalidad más extensiva. En consonancia con lo anterior, Dummett sostiene: "un concepto indefinidamente extensible es aquel que, si formamos una concepción definida de una totalidad todos cuyos miembros caen bajo ese concepto, podemos, por referencia a esa totalidad, caracterizar una totalidad más grande todos cuyos miembros caigan bajo él" (Dummett 1993, p. 441).

En esta misma línea, Fine presenta una versión del argumento que afecta a la cuantificación irrestricta. Supongamos que existe un cuantificador de primer orden " $\forall$ " para cuantificar sobre absolutamente todo lo que hay. Entonces, por el principio conjuntista de

${ }^{8} \mathrm{El}$ principio all-in-one establece que "cuantificar sobre ciertos objetos presupone que esos objetos constituyen una colección: una cosa de la cual los objetos son miembros". Cartwright (1994) critica el principio all-in-one, porque le parece inadecuada la idea de que "no podamos hablar de galletas en el bote a menos que constituyan un conjunto"; Parsons (1974) y Priest (2002), en cambio, lo defienden. Por otro lado, el artículo de Weir confronta los resultados de una semántica desarrollada sin el principio, con una que si lo tenga.

${ }^{9}$ En "Quantification without Domain", Uzquiano (en prensa) analiza el principio all-in-many. Cuantificar sobre todos los objetos que satisfacen una cierta condición, por ejemplo, ser un conjunto, ser un ordinal o ser idéntico a sí mismo, es presuponer que existe un dominio de objetos que satisfacen la condición, es decir, existen algunos objetos tales que un ítem es uno de ellos si y sólo si satisface la condición. Linnebo en "Pluralities and Sets" (inédito) defiende la idea de que siempre que haya una pluralidad de objetos es posible reunirlos a todos en un conjunto (objeto). 
separación, "podemos llegar a otra interpretación del cuantificador de acuerdo con la cual habrá un objeto cuyos miembros sean todos aquellos objetos, en el sentido (original) del cuantificador, que no son miembros de sí mismos" (p. 21). Por eso podemos introducir un nuevo cuantificador " $\forall+$ " que alcance este "nuevo" objeto también $y$, en consecuencia, darnos cuenta de que el presunto cuantificador absolutamente universal no lo era en absoluto.

De acuerdo con Fine, su versión del argumento no presupone una concepción de dominio como objeto. Particularmente, él cree que no utiliza el principio all-in-one. Donde hay cuantificación sobre ciertos objetos, el argumento de la extensibilidad muestra que debemos ser capaces de ir más allá y tomar estos objetos como una colección completa. Pero, ante este punto, surge una dificultad: ¿̨cómo podría expresarse la posición contraria a la cuantificación irrestricta si ningún cuantificador puede ser absolutamente general como para ser lo suficientemente expresivo y poder decir lo que se pretende decir? Aquí, Fine propone ir a lo modal: ante cualquier interpretación de un presunto cuantificador absolutamente universal, siempre será posible postular un dominio más grande de objetos que la extienda. Su versión modal del argumento de la extensibilidad abre una nueva e interesante línea que vincula temas de limitaciones expresivas con nociones modales. ${ }^{10}$ Por ejemplo, siempre será posible expresar una interpretación más inclusiva recurriendo a postulaciones modales que la que brinde cualquier cuantificación de primer orden. No me queda claro, sin embargo, que la posición de Fine evite el compromiso con el principio all-in-one. En tal caso, la posibilidad de desarrollar la semántica de un lenguaje por medio de un aparato teórico que evite la utilización de dominios de cuantificación quedaría inmune al argumento. Tampoco estoy seguro de que su apelación a modalidades sea explicativamente superior al posible recurso que en la misma dirección podría hacerse con plurales. Además, tengo dudas acerca de por qué el defensor de la cuantificación irrestricta no podría decir que su cuantificador de primer orden cubre cualquier posible postulación genuina que pudiera llegar a hacerse (no sólo cubre lo actual).

El tema del artículo de Shapiro y Wright, "All Things Indefinitely Extensible", son las totalidades indefinidamente extensibles. Los autores se centran en el universo conjuntista intentando esclarecer los principales aspectos de la cuantificación sobre conjuntos sin res-

\footnotetext{
${ }^{10}$ En una conversación con Øystein Linnebo, ambos discutimos la posibilidad de complementar el enfoque que presenta en esta compilación continuando la línea adoptada por Fine.
} 
tricción alguna. ¿Podemos hablar consistentemente sobre todos los conjuntos puros? o ¿sobre todas las entidades conjuntistas? Y en conexión con lo anterior, ¿podemos hablar de todos los números cardinales u ordinales? Estas preguntas remiten a los intentos de evitar todo recurso a multiplicidades inconsistentes, muchas veces apelando a algún tipo de limitación de tamaño dentro del dominio conjuntista. Los autores no ofrecen una respuesta a tales interrogantes, ya que "parece que toda [posición] tiene dificultades que serían suficientes para ser consideradas decisivas en contra de las mismas" (p. 293). Sin embargo, el trabajo tiene el valor de ofrecer una discusión completa en torno a esas dificultades con una esclarecedora caracterización de la mayor parte de los principales aspectos ligados a las totalidades indefinidamente extensibles.

A lo largo del artículo aparece la conjetura de Russell, según la cual un concepto es indefinidamente extensible si y sólo si existe una inyección entre los ordinales y sus instancias. Los autores brindan un argumento a favor de la misma (que usa una inducción transfinita sobre los ordinales). No obstante, ambos piensan que la conexión entre extensibilidad indefinida y la existencia de la mencionada función uno a uno no es suficiente para elucidar la noción, particularmente, porque no nos explica por qué el concepto de ordinal es indefinidamente extensible. Para los autores, en la caracterización de los conceptos indefinidamente extensibles es crucial el reconocimiento de cierta relativización: el concepto $X$ es indefinidamente extensible con respecto a $Y$. El caso más extremo es el del concepto de conjunto que resulta ser indefinidamente extensible con respecto al concepto mismo de conjunto, en el sentido de que para todo conjunto de conjuntos hay un conjunto que no está en ese conjunto. Éste es uno de los motivos para notar que bajo esta concepción no existe el conjunto de todos los conjuntos.

En "Unrestricted Unrestricted Quantification: The Cardinal Problem of Absolute Generality", Uzquiano retoma los temas de expresividad vinculados a la posibilidad de formular generalidad absoluta por medio de recursos que provengan de la adopción de lenguajes plurales o de orden superior. El recurso a tales mecanismos entra en conflicto con ciertas restricciones incompatibles con el tamaño del universo. En el artículo de Uzquiano se presentan dos ejemplos distintos: contradicciones que surgen a partir de la admisión de cuantificación absolutamente irrestricta, o cuando ciertos principios mereológicos o de la teoría general de la abstracción se aplican a la teoría de conjuntos $\mathrm{ZF}$ con urelementos y cuantificadores plurales o de orden superior 
(ZFCSU). En ambos casos, Uzquiano hace notar que la totalidad de todos los conjuntos debe ser de tamaño fuertemente inaccesible, pero los principios mereológicos plausibles implican que la totalidad es del mismo tamaño que el resultado de aplicar una operación análoga a la de conjunto potencia a cierta totalidad y, por lo tanto, no es inaccesible.

Algo similar sucede con la teoría general de la abstracción. Lo que muestra el argumento de Uzquiano es que la cardinalidad del universo es tanto accesible como inaccesible. Según su posición, parece difícil encontrar una solución unificada que no pague un alto costo por preservar los máximos recursos expresivos. ¿Podríamos dejar de suponer que los individuos forman siempre un conjunto? ¿O quizá no todos los objetos puedan entrar en relaciones de pertenencia? ¿Habrá que hacer cambios drásticos en la mereología extensional?

\section{IV}

Quizá tengamos que explorar si no existe un mecanismo más básico que la cuantificación para lograr absoluta generalidad. En esta dirección, Williamson en "Absolute Identity and Absolute Generality", y McGee en "There's a Rule for Everything" proponen una comprensión de los cuantificadores vinculada a la textura abierta (open ended) de las reglas de inferencia. Williamson propone usar la absoluta identidad para entender la absoluta generalidad: "dada una noción apropiada de validez [...], tanto la regla de introducción del cuantificador universal $[\forall$-Introduction], como la de eliminación $[\forall$ Elimination] son válidas en la lectura irrestricta del cuantificador" (p. 383) De esta manera, según el autor, los cuantificadores de primer orden se pueden interpretar legítimamente como si hablaran de absolutamente todo lo que hay. En la misma línea, McGee argumenta a favor de una lectura inferencialista de los cuantificadores para asegurar absoluta generalidad aun en un universo en expansión indefinida. El autor apela al Teorema de Harris, que garantiza la unicidad de los cuantificadores a partir de su interderivabilidad. Si bien este teorema no alcanza para mostrar que no hay otros usos legítimos de " $\forall$ " además de su uso irrestricto, muestra que diferentes modos de usar esta expresión no pueden convivir sin generar problemas. McGee afirma "el Teorema de Harris [...] nos da una razón para anticipar que, cuando desarrollamos una teoría semántica, se favorecerá la cuantificación desambiguamente irrestricta [unambiguously unrestricted quantification]" (p. 193). 
En "Something about Everything: Universal Quantification in the Universal Sense of Universal Quantification", Lavine propone lograr toda la generalidad posible usando esquemas, en lugar de cuantificadores. En lugar de Absolute unrestricted generality, full schematic generality. Los esquemas no son reducibles a la cuantificación. De acuerdo con Lavine, hay un modo distinto de expresar generalidad usando esquemas, para declarar que "cualquier [any] instancia (tiene cierta propiedad), donde 'cualquier' tiene que distinguirse claramente de "toda' [every]" (p. 118). De hecho, Lavine va más allá al hablar de un tipo de generalidad involucrada en los esquemas que resulta más primitiva que la generalidad cuantificacional. Sin embargo, la posición de Lavine me despierta dudas: no es fácil entender en qué sentido la generalización por la vía de esquemas es algo distinto de la generalización por medio de cuantificador. Más allá de este punto, y vinculado con la interpretación del Axioma de Inducción de la Aritmética, Shapiro (2005a) argumenta, contrastando las lecturas

$$
\begin{array}{ll}
\text { Axioma cuantificado } & (\forall P)((P 0 \& \forall x(P x \rightarrow P s x)) \rightarrow \forall x P x) \\
\text { Axioma esquema } & ((\boldsymbol{P} 0 \& \forall x(\boldsymbol{P} x \rightarrow \boldsymbol{P} s x)) \rightarrow \forall \mathrm{x} \boldsymbol{P} x)
\end{array}
$$

que los esquemas no alcanzan a expresar la idea intuitiva según la cual ciertos objetos matemáticos comparten cierta propiedad. Esto es así, ya que la lectura en orden superior es categórica (es verdadera sólo en modelos cuyos dominios son los números naturales estándar), mientras que el uso de esquemas no es inmune a la skolemización. Sin embargo, McGee tiene una posición distinta de la de Shapiro (McGee 1997, p. 59). Desde su punto de vista, los esquemas (openended schemas) ofrecen un modo de obtener categoricidad sin pagar los costos ontológicos de adoptar cuantificación de orden superior. Aunque, claro, su posición ha recibido críticas. Pedersen y Rossberg sostienen que, contrario a lo que argumenta McGee, "los esquemas [open-ended] y la lógica de segundo orden están parejos (expresivamente), cuando toman compromisos ontológicos (Pedersen y Rossberg inédito, p. 59).

Como hemos visto, al costado ontológico de la tesis de la absoluta universalidad subyace la existencia de una "todalidad absolutamente inclusiva". Sin embargo, a partir de los trabajos de Carnap y de Quine han surgido todo tipo de cuestionamientos escépticos a las 
posiciones ontológicas que intentan responder preguntas denominadas externas. ${ }^{11}$ La pregunta por lo que hay es siempre interna y por razones metaontológicas no existen condiciones correctas para responder preguntas externas. Claro que lo que desea el defensor de la cuantificación absolutamente irrestricta es el empleo de la cuantificación universal en contextos trans-esquema conceptual y transortal. No obstante, el planteamiento de Carnap-Quine es que hay una serie de problemas metaontológicos vinculados a la noción de todos los objetos.

En su artículo "Against "Absolutely Everything", Hellman se enfoca en la posibilidad de ontologías alternativas fácticamente equivalentes: "la misma situación fáctica subyacente puede describirse adecuadamente y con bastante precisión en diversos modos ontológicos. Sería arbitrario y sin garantías decir que uno es "realmente correcto" (p. 83). Por eso, no existe modo de que el absolutista pueda asegurar que está cuantificando sobre absolutamente todo lo que hay. A diferencia de Glanzberg, Hellman se concentra en los aspectos metaontológicos de la disputa. Sólo garantizando que existe una respuesta para la pregunta externa en metaontología, el absolutista bloquearía el argumento. Pero si, siguiendo a Carnap, pensamos que no existe posibilidad de dar respuestas a las preguntas externas, el absolutista parece estar en problemas. Ahora bien, al igual que Glanzberg, Hellman sugiere una interpretación contextualista de las presuntas generalizaciones absolutamente irrestrictas, las cuales se toman como si contuvieran un elemento esquemático.

Mientras que el absolutista sostiene que hay un único dominio fijo, el cual es simplemente "absolutamente todo", el enfoque contextualista que presenta Glanzberg en "Context and Unrestricted Quantification" implica que diferentes contextos pueden tener diferentes dominios subyacentes. El principal propósito del artículo es comprender mejor las restricciones contextuales de la cuantificación surgidas a partir de las paradojas. Su planteamiento principal es que los cuantificadores siempre tienen que ser comprendidos como si hablaran contextualmente de un dominio. Y lo que paradojas como las de Russell muestran es que "para cualquier contexto dado, hay otro distinto que brinda un dominio más amplio de cuantificación" (p. 47). Por ese motivo, su posición es defender una versión contextualista del enfoque de que no hay absolutamente cuantificación irrestricta. En este sentido, argumenta que es posible especificar los principios generales que rigen la construcción de nuevos dominios. Es

${ }^{11}$ Rayo defiende una posición neocarnapiana en Rayo 2007. 
importante destacar la discusión incluida en el artículo de Glanzberg sobre cuán lejos debemos iterar el proceso de expansión de los dominios. Usualmente se piensa que no hay un límite, pero Glanzberg, en contraste, sostiene que existen razones para incluir la iteración justo hasta el primer ordinal no recursivo.

Otro aspecto vinculado a la relativización ontológica es la posibilidad de que exista cierta indeterminación de tal cuantificación absolutamente irrestricta como producto del Teorema LöwenheimSkolem. Los artículos de Lavine, McGee y Williamson tratan esta dificultad. Conforme a este resultado, las teorías de primer orden tienen modelos estándar y no estándar. Por ejemplo, la aritmética de Peano formulada en primer orden tiene modelos cuyos dominios incluyen elementos no estándar. Por eso, en contra de la absoluta generalidad, el skolemita sostiene que cuando se habla de números naturales se podría estar hablando de "otros" objetos que no sean los números naturales estándar. Y el tema siempre puede generalizarse: dada cualquier teoría de primer orden en la que se intentara por medio de sus cuantificadores hablar de todo lo que hay, siempre será posible encontrar otro modelo que incluya "otros" elementos. Sin embargo, esta indeterminación sólo afecta a los lenguajes de primer orden: para lenguajes de mayor poder expresivo cuyos cuantificadores sean de orden superior no se aplica tal indeterminación.

\section{VI}

Los lenguajes son creaciones humanas y no parece haber límites a lo que se pueda llegar a decir. Los bordes del proceso no están dados de antemano y ante cualquier frontera se da la posibilidad de trascenderla. Absolute Generality nos desafía a evitar el pesimismo semántico: la tesis según la cual hay interpretaciones que no pueden expresarse ni siquiera adoptando cuantificadores frente a los que presumimos máxima generalidad. Todo tipo de jerarquías abiertas parecen surgir: o jerarquías de plurales, o de órdenes de cuantificadores, o extensiones de conceptos semánticos que sólo pueden expresarse desde un lenguaje con mayor poder expresivo. Luego de la lectura del libro objeto de esta nota, no estoy convencido de que puedan funcionar los mecanismos ofrecidos como solución a los problemas de la absoluta generalidad. ¿Cómo evitar, entonces, adoptar algún tipo de pesimismo? Esto es, que haya lenguajes cuya semántica no sea expresable, o que haya objetos que queden fuera del alcance de nuestros cuantificadores. Adoptar algún tipo de estrategia extensibilista negando la opción de que sea posible la unión sobre todos los lenguajes 
parece una de las opciones programáticas. Tampoco debe descartarse la posibilidad de que debilitando la lógica podamos alcanzar máxima expresividad. En ambas estrategias, se evita el pesimismo, ya que no hay nada que esté más allá de lo que pueda expresarse, aunque quizá nunca se pueda abarcar todo completamente. Absolute Generality nos enseña a tolerar la decepción de no entender de manera cabal cómo podemos hablar de absolutamente todo lo que hay sin caer en un profundo pesimismo expresivo.

\section{BIBLIOGRAFÍA}

Cartwright, R., 1994, "Speaking of Everything", Nô̂s, vol. 28, no. 1, pp. 120.

Dieveney, P., 2008, Review on Absolute Generality, Mind, vol. 117, no. 467, pp. 719-722.

Dummett, M., 1993, The Seas of Language, Oxford University Press, Oxford.

—_, 1991, Frege: Philosophy of Mathematics, Harvard University Press, Cambridge, Mass.

Field, H., 2008, Saving Truth from Paradox, Oxford University Press, Oxford.

García de la Sienra, A. (comp.), 2008, Reflexiones sobre la paradoja de Orayen, Instituto de Investigaciones Filosóficas-UnAM, México.

Linnebo, Ø., 2003, "Plural Quantification Exposed", Noûs, vol. 37, no. 1, pp. 71-92.

Maudlin, T., 2004, Truth and Paradox, Oxford University Press, Oxford.

McGee, V., 1997, "How We Learn Mathematical Language", The Philosophical Review, vol. 106, no. 1, pp. 35-68.

Moretti, A. y G. Hurtado (comps.), 2003, La Paradoja de Orayen, Eudeba, Buenos Aires.

Orayen, R., 2003, "Una paradoja en la semántica de la teoría de conjuntos", en Moretti y Hurtado 2003, pp. 35-59.

Parsons, C., 1974, "Set and Classes", Noûs, vol. 8, no. 1, pp. 1-12.

Pedersen, N. y M. Rossberg, inédito, aceptado para su publicación en H. Bohse (comp.), Selected Papers Contributed to the Sections of GAP.6, Sixth International Congress of the German Society for Analytical Philosophy.

Priest, G., 2007, "Review of Absolute Generality", Notre Dame Philosophical Reviews, <http://ndpr.nd.edu/review.cfm?id=11144> [17/09/2007].

— 2006, In Contradiction, Oxford University Press, Oxford.

, 2002, Beyond the Limits of Thought, Cambridge University Press, Cambridge.

Rayo, A., 2007, "Ontological Commitment", Philosophy Compass, vol. 2, no. 3 , pp. $428-444$. 
Rayo, A., 2005, "La Paradoja de Orayen", Crítica, vol. 37, no. 109, pp. 99-115.

Resnik, M., 1988, "Second-Order Logic Still Wild", The Journal of Philosophy, vol. 85, no. 1, pp. 75-87.

Shapiro, S., 2005a, "Higher-Order Logic", en Shapiro 2005b, pp. 751-780.

Shapiro, S. (comp.), 2005b, The Oxford Handbook of Philosophy of Mathematics and Logic, Oxford University Press, Nueva York.

Smith, P., 2008, "Review on Absolute Generality", The Bulletin of Symbolic Logic, vol. 14, no. 2, pp. 398-401.

Tarski, A., 1956 (1990), Logic, Semantics and Metamathematics, 2a. ed., Oxford University Press, Oxford.

_ 1935, "The Concept of Truth in Formalized Languages", en Tarski 1956, pp. 152-278.

Uzquiano, G., en prensa, "Quantification without Domain", en O. Bueno y Ø. Linnebo (comps.), New Waves in the Philosophy of Mathematics, Palgrave Macmillan, Basingstoke.

Weatherson, B., Thoughts Arguments and Rants, en <http://tar.weatherson. org $/>[5 / 01 / 2005]$.

Williamson, T., 2003, "Everything", Philosophical Perspectives, vol. 17, pp. 415-465.

Recibido: 21 de octubre de 2008; revisado: 9 de noviembre de 2008; aceptado: 16 de noviembre de 2008. 